Santa Clara University

Scholar Commons

Physics

College of Arts \& Sciences

3-6-2001

\title{
Crossover from phase fluctuation to amplitude- dominated superconductivity: A model system
}

Richard P. Barber Jr.

SantaClara University, rbarber@scu.edu

L. Merchant

J. Ostrick

Robert C. Dynes

Follow this and additional works at: http://scholarcommons.scu.edu/physics

Part of the Physics Commons

\section{Recommended Citation}

L. Merchant, J. Ostrick, R. P. Barber, Jr. and R. C. Dynes, "Crossover from phase fluctuation to amplitude-dominated superconductivity: A model system," Phys. Rev. B., 63, 134508, (2001).

This Article is brought to you for free and open access by the College of Arts \& Sciences at Scholar Commons. It has been accepted for inclusion in

Physics by an authorized administrator of Scholar Commons. For more information, please contact rscroggin@scu.edu. 


\title{
Crossover from phase fluctuation to amplitude-dominated superconductivity: A model system
}

\author{
L. Merchant, ${ }^{1}$ J. Ostrick, ${ }^{1}$ R. P. Barber, Jr., ${ }^{2}$ and R. C. Dynes ${ }^{1}$ \\ ${ }^{1}$ Department of Physics, University of California, San Diego, La Jolla, California 92093 \\ ${ }^{2}$ Department of Physics, Santa Clara University, Santa Clara, California 95053
}

(Received 9 October 2000; published 6 March 2001)

\begin{abstract}
We have experimentally studied a model system that demonstrates the crossover from a superconductor that is dominated by phase fluctuations, to one in which the amplitude of the order parameter is the controlling influence on $T_{c}$. This model system is comprised of two-dimensional granular $\mathrm{Pb}$ with an overlayer of $\mathrm{Ag}$. The system displays many aspects of the phase diagram of the concentration dependence of $T_{c}$ in the high- $T_{c}$ superconductors, and this crossover has been applied to explain the phase diagram in that case. We point out the similarities and differences between the model system presented in this paper and the high- $T_{c}$ superconductors.
\end{abstract}

DOI: 10.1103/PhysRevB.63.134508

PACS number(s): 74.40.+k, 74.76.-w, 74.80.- $-\mathrm{g}$

\section{INTRODUCTION}

Superconductivity is reasonably well understood in conventional materials. BCS theory and the strong coupling modifications have been quite successful in elucidating the basic mechanisms and the variety of observed phenomena associated with the long-range phase coherence of the order parameter. ${ }^{1}$ The order parameter can be written $\psi=\psi_{0} e^{i \phi}$, where $\psi_{0}$ (the amplitude) determines quantities such as the energy gap $(\Delta)$ and the transition temperature $T_{c}$. The phase $(\phi)$ and the phase stiffness determine the superconductor's ability to carry a supercurrent. For example, the supercurrent density $J_{s}$ varies as the gradient of the phase

$$
J_{s} \alpha \nabla \phi .
$$

A particularly illuminating example of this is illustrated in the Josephson relations between two superconductors that are weakly coupled, where the dc Josephson current is given by $J_{c}=J_{0} \sin \theta . J_{0}$ is the maximum allowed supercurrent and $\theta$ is the phase difference $\left(\phi_{2}-\phi_{1}\right)$ between the two superconductors. For weak currents and hence small phase differences $\sin \theta \approx \theta$ and the Josephson relation reduce to the BCS relation $J=J_{0} \nabla \phi$.

Superconductivity can then be destroyed by either a suppression of the amplitude of the order parameter $\left(\psi_{0}\right.$ and hence $\Delta$ and $T_{c}$ go to zero) or a fluctuation in the phase locking resulting in a time dependence to $\phi$ or $\theta$. The timedependent Josephson relation is given by $\dot{\phi}=2 \mathrm{eV} / \hbar$ and so a time dependence in $\phi$ results in a voltage. Trivial examples of the two cases in conventional superconductors would be (a) raising the temperature above $T_{c}$, resulting in $\Delta$ going to zero and (b) increasing the supercurrent above its critical current for vortex generation and propagation, resulting in a time-dependent phase and thus a voltage.

Recently, models for understanding observations in the high- $T_{c}$ superconductors have suggested that considerations of phase and amplitude suppression in the order parameter are particularly relevant. Specifically, it has been suggested that the familiar phase diagram for superconductivity (shown schematically in Fig. 1) can be understood as a crossover from a $T_{c}$ dominated by phase fluctuation in the low- concentration regime to amplitude dominated behavior at the high end. ${ }^{2}$ In this picture at low-carrier concentration, the material spontaneously decomposes into an electronically spatially inhomogeneous material and the superconducting order parameter follows this inhomogeneity. The stiffness of the order parameter phase weakens locally, becomes more susceptible to fluctuations, and driven by thermal fluctuations, the measured ${ }^{3} T_{c}$ drops for a decreasing concentration of dopants. With increasing concentration the $T_{c}$ reaches a maximum. Above the maximum or optimal $T_{c}$, the amplitude of the order parameter is reduced with increasing carrier concentration (a microscopic model for this effect that is generally accepted is not yet available). In this picture the "optimal $T_{c}$ " is simply a crossover from the phase dominant behavior to the amplitude dominated $T_{c}$. A consequence of this picture is illustrated in Fig. 1 where on the lowconcentration side, while $T_{c}$ is suppressed by phase fluctuations, signatures of the amplitude of the order parameter should be observable at temperatures well above the measured ${ }^{3} T_{c}$. This model has been used by some to explain
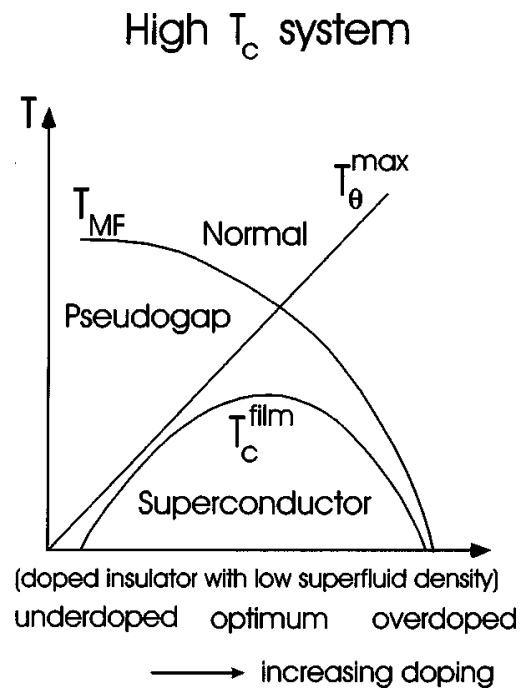

FIG. 1. A model phase diagram for the high- $T_{c}$ superconductors as a function of doping. $T_{\theta}$ is the phase fluctuation limiting $T_{c}, T_{\mathrm{MF}}$ is the amplitude limiting mean-field $T_{c}$. 
the observed pseudogap at temperatures higher than $T_{c}$ on the low-doping side.

In this paper we describe a "model" system that we have studied to probe the phase diagram implied by this picture of phase and amplitude domination of the order parameter. We have chosen a two-dimensional (2D) random "granular" array of a conventional superconductor wherein we continuously tune the coupling between the grains so that we can study the equivalent phase diagram of Fig. 1. We begin on the left-hand side of the figure where $T_{c} \rightarrow 0$ and the system is, in fact, insulating. We then continuously enhance the coupling between the grains. In this region, each grain is independently superconducting but their individual phases $\left(\phi_{1}, \phi_{2}, \ldots, \phi_{n}\right)$ are weakly coupled. Hence superconductivity in this regime is governed by the phase fluctuations in the system. With increasing coupling, the phases finally strongly lock and we cross over to a superconductor whose order parameter amplitude dominates.

\section{EXPERIMENT}

The system we have investigated is quench condensed granular Pb. This system has been studied previously, ${ }^{4}$ and it has been demonstrated that as a function of "mean thickness" the system can be driven through the superconductorinsulator transition for a microscopic sheet resistance in the vicinity of $R_{\square} \approx \hbar / 2 e^{2}$. It has been shown from tunneling measurements ${ }^{5}$ that on the insulating side of the superconductor insulator transition, each grain is separately and independently superconducting while a transport measurement shows the film to be insulating.

The experiments have been performed in a specially designed dilution refrigerator and in a pumped ${ }^{4} \mathrm{He}$ cryostat. The films were grown in situ in a vacuum chamber located inside the cryostat. The vacuum chamber was completely surrounded by ${ }^{4} \mathrm{He}$ liquid and by pumping the chamber and via the natural cryopumping, the evaporations were performed in a UHV environment. Leads were attached to the substrate (glass or $\mathrm{Si}_{-} \mathrm{SiO}_{2}$ ) so that the film could be continuously grown; and at a chosen thickness, growth was terminated and the film studied. Tunnel junctions were fabricated using the same film on which transport measurements were performed. The counterelectrode, this $\mathrm{Al}-\mathrm{Al}_{2} \mathrm{O}_{3}$-granular $\mathrm{Pb}$ tunnel junction, was produced by first depositing an $\mathrm{Al}$ film in a separate chamber. ${ }^{5,6}$

\section{RESULTS AND DISCUSSION}

After transport and tunneling measurements for each thickness, growth was resumed. During growth, the substrate was held at $10 \mathrm{~K}$. In Fig. 2 we show a schematic of the morphology of the granular film of this nature as studied by in situ scanning tunnel microscopy (STM), ${ }^{7}$ and in the lower portion of the figure a typical example of a data set for granular $\mathrm{Pb}$ films. Here we show $\log R$ vs $T$ for a film grown sequentially with a resistance at $T=10 \mathrm{~K}$ from $10^{6} \Omega / \square$ to $10^{1} \Omega / \square$. For the thinnest film, the grains are weakly coupled and the resistance shows insulating activated behavior. We will show that in this case each grain is supercon-

\section{Granular Morphology}
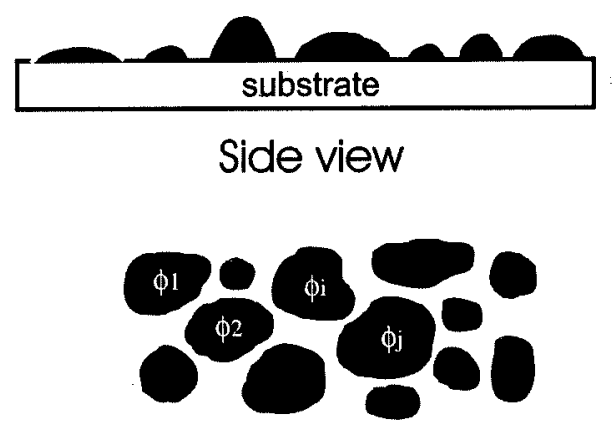

Top view

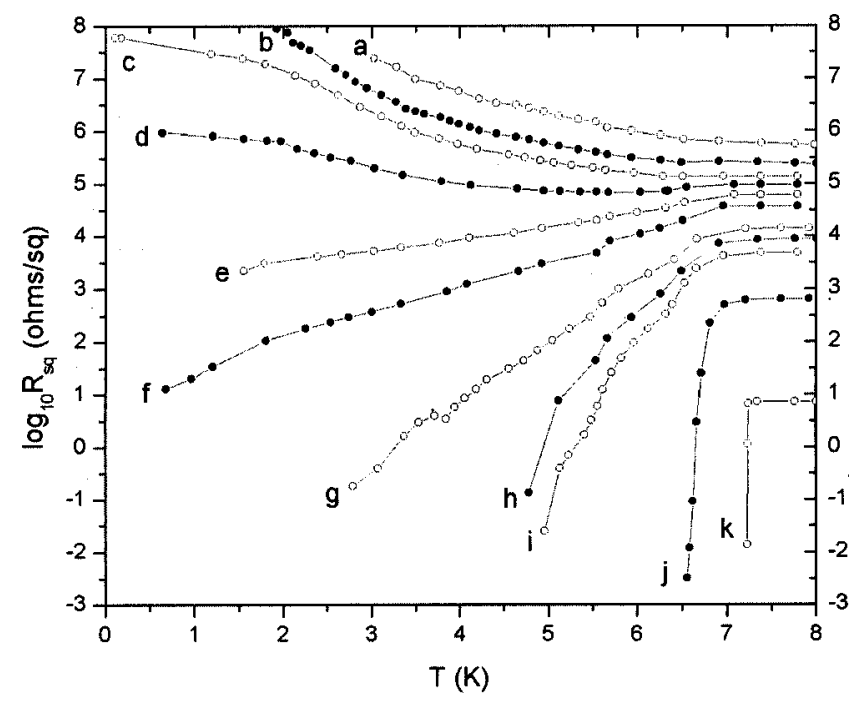

FIG. 2. Two-dimensional sheet resistance transport of a granular $\mathrm{Pb}$ film. The upper part of the figure illustrates the morphology. Each grain is individually superconducting with its own phase $\phi_{i}$.

ducting (the amplitude of the order parameter is well defined); but the grains are electrically connected only via hopping or tunneling, so the grains are dephased as schematically illustrated in the cartoon in Fig. 2. The insulating behavior is consistent with activated conduction. It is interesting to note that below the transition temperature $T_{c}$ of bulk $\mathrm{Pb}$, the activation energy increases by approximately a value equal to the superconducting energy gap $\Delta$ $=1.4 \mathrm{meV} \cdot{ }^{4,5}$ Our physical picture is that conduction is either by tunneling or activation; and if tunneling between the grains is significant, the conduction is similar to a series/ parallel array of superconductor insulator-superconductor (SIS) tunnel junctions. In that case, the resistance at low bias (below $\Delta$ ) indeed increases exponentially with an activation energy $\Delta$.

Examination of Fig. 2 illustrates some interesting features. In the region of resistance $10^{4}-10^{5} \Omega / \square$ there is a transition from insulating behavior to behavior that appears as if superconducting fluctuations begin to suppress the resistance. ${ }^{4}$ With decreasing temperature, the resistance con- 
tinues to decrease but there is no clear superconducting transition where $R=0$. For the cases near the $S-I$ transition (curves $e, f$, and $g$ for example), it is not clear that $R$ will go to zero as we approach $T=0$. We have previously studied this curious behavior in several superconductors 8 to a temperature as low as $50 \mathrm{mK}$, where the resistance continues to follow this trend (for example, curve $f$ in Fig. 2). Oddly, it appears as if the resistance exhibits a form

$$
R_{\square}=R_{0} e^{T / T_{0}} .
$$

One can imagine a qualitative physical picture in this regime where the length scale for phase coherence and phase locking increases with decreasing temperature but in order that the $T=0$ intercept remain finite $\left[R_{0}\right.$ in Eq. (1)], we must invoke quantum fluctuations. While this regime does not exactly match the low-doping side of the phase diagram of the high- $T_{c}$ superconductors as shown in Fig. 1, the similarities and differences are worth underlining. With increased coupling between the grains, the system is more inclined to be globally superconducting. This comes about because the phase $\phi$ of the global order parameter becomes stiffer in the superconductor and the phase fluctuations attempting to destroy the long-range order are suppressed. Likewise in the phase fluctuation interpretation in Fig. 1 of the rising $T_{c}$ with increasing dopant concentration, the physical picture is that the phase becomes stiffer, ${ }^{2}$ thus enhancing $T_{c}$ where longrange global phase locking occurs. The difference in the two cases is that it is believed that in the high- $T_{c}$ case, the material truly becomes superconducting, while in the granular case, quantum fluctuations apparently destroy the long-range coherence down to the lowest measured temperatures. If this analogy is valid, the two dimensional nature of the conventional granular superconductor as opposed to the "quasi" two dimensions of the high- $T_{c}$ superconductors could explain the difference.

A remarkable similarity in the two cases is the observation that at a temperature well above the long-range superconducting transition $T_{c}$, an energy gap or pseudogap is observed. This has been extensively studied in the high- $T_{c}$ case and continues to be a subject of interest and controversy. In the case studied in this paper, the observation of an energy gap is quite striking and understandable as the individual grains themselves are superconducting at the bulk $T_{c}$ for $\mathrm{Pb}$. The observation of an energy gap (or "pseudogap") in the granular $\mathrm{Pb}$ case is shown in Fig. 3. In this figure we plot the $I-V$ characteristics of an $\mathrm{Al}^{-} \mathrm{Al}_{2} \mathrm{O}_{3}$ - granular $\mathrm{Pb}$ tunnel junction for three of the samples $(b, h$, and $k)$ of Fig. 2 . The three tunneling curves are very similar and illustrate very clearly the characteristic current rise at the energy gap of $\mathrm{Pb}(1.4$ $\mathrm{meV}) .{ }^{5} \mathrm{In}$ all three cases, current is suppressed until a bias of $\mathrm{eV} \sim \Delta$ is applied at which point states are available above the energy gap and current is allowed to flow. The striking feature about these three curves is that sample $b$ is an insulator and $h$ and $k$ are probably superconducting at $2.1 \mathrm{~K}$. If they are not, the resistance is well below our measurement capabilities and at least 20 orders of magnitude below sample $b$. In analogy with the high- $T_{c}$ situation, we would argue that sample $b$ (and several others such as $a, c, d, e, f$,

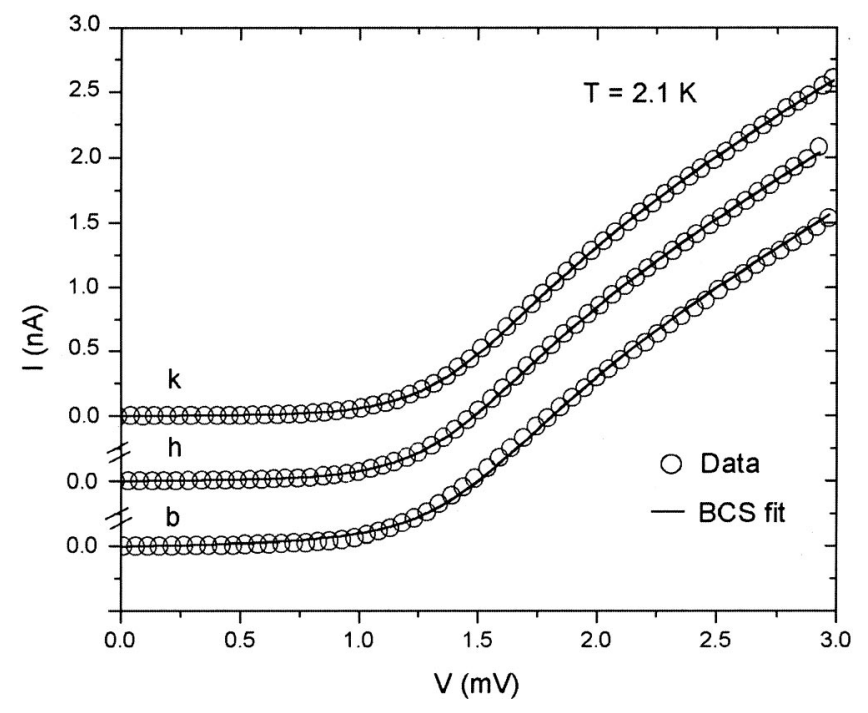

FIG. 3. The $I-V$ characteristics for Al-insulator-granular $\mathrm{Pb}$ tunnel junctions at $T=2.1 \mathrm{~K}$ for the samples $b, h$, and $k$ of Fig. 2 . The energy gap $\Delta$ of $\mathrm{Pb}$ is observed in all three curves despite the fact that $h$ and $k$ are superconductors and $b$ is an insulator.

etc.) have a pseudogap well above any superconducting transition. In this case it is straightforward to understand that as the gap measures the amplitude $\psi_{0}$ of the order parameter, the transport is a probe of the phase $\phi$ and how it locks. In this case the amplitude follows conventional superconducting wisdom, and begins to open at the "conventional" $T_{c}$ of 7.2 K. The phase fluctuations can be sufficiently severe to result in nonsuperconducting and even insulating behavior.

We have created a model system that allows us to not only continuously enhance the phase coupling as illustrated in Fig. 2, but to go over the optimum peak of Fig. 1 and study the regime where superconductivity is dominated by the amplitude of the order parameter. This system allows us to map out and study a phase diagram illustrated in Fig. 1 with the distinction that we have already raised in the discussion of Fig. 2; it is not clear in the data of Fig. 2 whether or not we can define a superconducting transition for several of the samples. If the critical behavior follows a

$$
R=R_{0} e^{T / T_{0}}
$$

behavior to $T=0$, dissipation persists to $T=0$. This exponential behavior can be deceptive, however, as illustrated in Fig. 4. Here we show curves $e, f$, and $g$ from Fig. 2 where the resistance is plotted on a linear scale. By plotting the data this way, one could be led to believe that there is a superconducting transition; and by choosing to define $T_{c}$ at the point where $R=R_{N} / 2$, one would conclude that $T_{c}$ decreases with increasing $R_{\square}$ as the grains decouple. Here $R_{0}$ is sufficiently low that it is difficult to observe on this linear scale. This conclusion would clearly obscure the exponential behavior of Fig. 2 and result in the simpler interpretation that the resistive transition is broadened. Such a conclusion is clearly in error.

The model system that allows us the full range of Fig. 1 is schematically illustrated at the top of Fig. 5 and the results of such a study are illustrated in the same figure. We begin with 


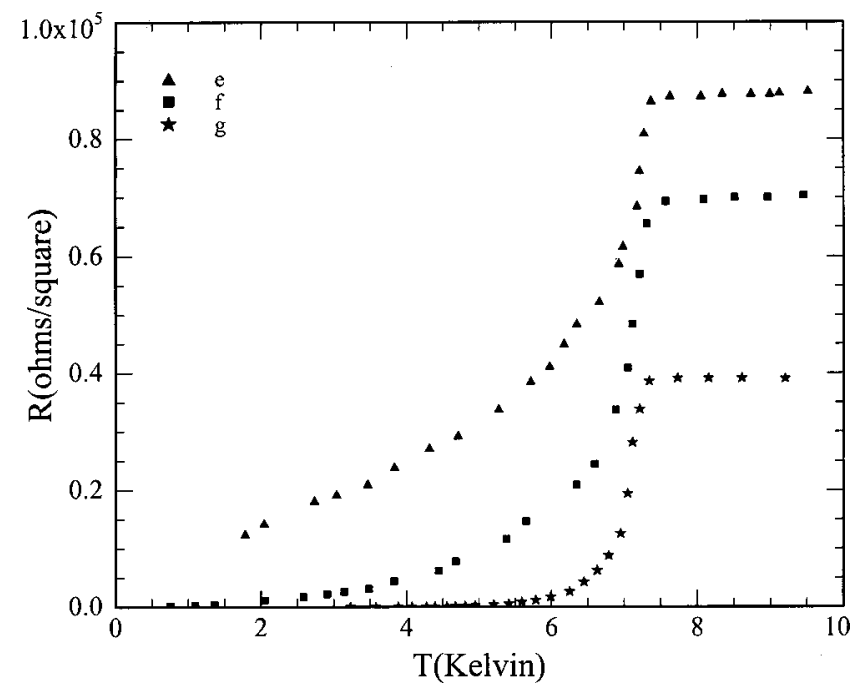

FIG. 4. Sheet resistance as a function of temperature for samples $e, f$, and $g$ of Fig. 2. These data are shown on a linear plot and illustrate how the $e^{T}$ behavior observed in Fig. 2 can be obscured.

an insulating film of granular $\mathrm{Pb}$, quench condensed on a substrate held at $10 \mathrm{~K}$. This film is labeled curve $a$ in Fig. 5 and is clearly insulating. From the previous discussion we know that the individual $\mathrm{Pb}$ grains are independently superconducting. Subsequent depositions on the random array of $\mathrm{Pb}$ grains are from a $\mathrm{Ag}$ source, not $\mathrm{Pb}$. The $\mathrm{Ag}$ allows us to scan through the phase diagram of Fig. 1. Originally, the Ag tends to strengthen the tunneling conductance between $\mathrm{Pb}$ grains in a similar fashion that additional $\mathrm{Pb}$ would. As the distance between metallic grains decreases, the resistance drops (exponentially) until the grains begin to become Jo-
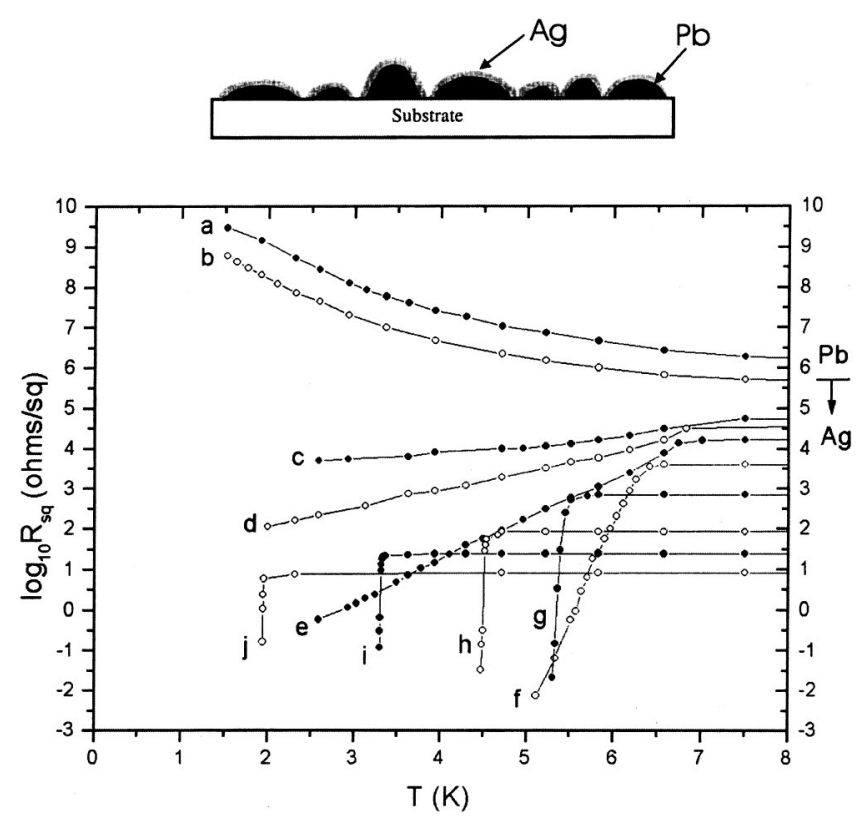

FIG. 5. Sheet resistance transport for granular $\mathrm{Pb}$ overlaid with $\mathrm{Ag}$. The $\mathrm{Pb}$ is originally insulating (curves $a$ and $b$ ) and then with the addition of Ag the system becomes superconducting. The crossover from phase fluctuation dominated to amplitude dominated superconductivity occurs in the range $e \rightarrow f \rightarrow g$.

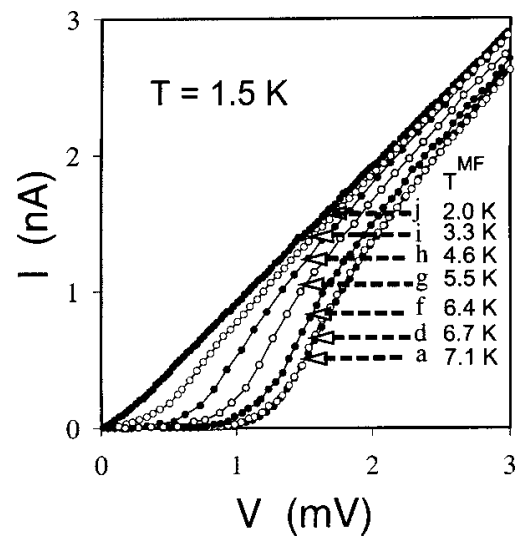

FIG. 6. $I-V$ characteristics at $T=1.5 \mathrm{~K}$ for tunneling into samples of Fig. 5. Listed also is the mean-field transition temperature where the energy gap $\Delta$ begins to open. This is the $T_{c}$ for the amplitude of the order parameter. For sample $a$ and $d$ this measures the "pseudogap."

sephson coupled. Curves $b, c, d, e$, and $f$, for example, illustrate behavior very similar to that shown in Fig. 2. For this case, however, it is the Ag that is stiffening the phase coupling between grains and inducing superconductivity in the array through SNS Josephson coupling ( $N$ is a normal metal). ${ }^{9}$ With increasing Ag, the " $T_{c}$," increases and in analogy with Fig. 1, it can be thought of as increasing the $T_{c}$ on the rising side of the curve as a function of concentration (in this case, Ag coupling of the grains). However, with even further increases in the thickness of Ag, it can be seen in Fig. 5 that the " $T_{c}$ ", begins to decrease (curves $g, h, i$, and $j$ ). This decrease comes about as a result of the proximity effect $^{10}$ of $\mathrm{Ag}$ on $\mathrm{Pb}$. Except for the thickest films (curve $j$, for example) the mean thickness of all the films in this study is very small $(\$ 10 \mathrm{~nm})$ and so these studies are all in the Cooper limit. ${ }^{11}$ In this limit one can think of the electrons experiencing an average pairing interaction (average of the two constituents). If we think of superconductivity in the BCS limit, the $T_{c}$ can be written

$$
k T_{c}=1.13 \hbar \omega_{D} e^{1 / N(0) V},
$$

where $\omega_{\mathrm{D}}$ is the debye frequency and $N(0) V$ is the net pairing interaction $(N(0)=$ the density of states at the fermi level and $V$ the pairing interaction). In the deGennes model ${ }^{10}$ for the proximity effect in the Cooper limit, the resultant pairing interaction for a superconducting $(S)$ and normal $(N)$ material in proximity is given by

$$
[N(0) V]_{S+N}=\frac{d_{S}}{d_{S}+d_{N}}[N(0) V]_{S},
$$

where $d_{S, N}$ is the thickness of the superconducting, normal metal layer and it is assumed that the pairing interaction in the normal metal $\left(V_{N}\right)$ is negligible. This simple "geometric mean" assumes that the electrons sample the normal metal and the superconducting metal equally in a coherence volume. Hence, with increasing $\mathrm{Ag}$ thickness $d_{N}$ the $T_{c}$ continues to reduce to a vanishingly small value (in the Cooper limit). Further evidence for this reduction and that these 


\section{(a) Initial insulating $\mathrm{Pb}$ film $+\mathrm{Ag}$}
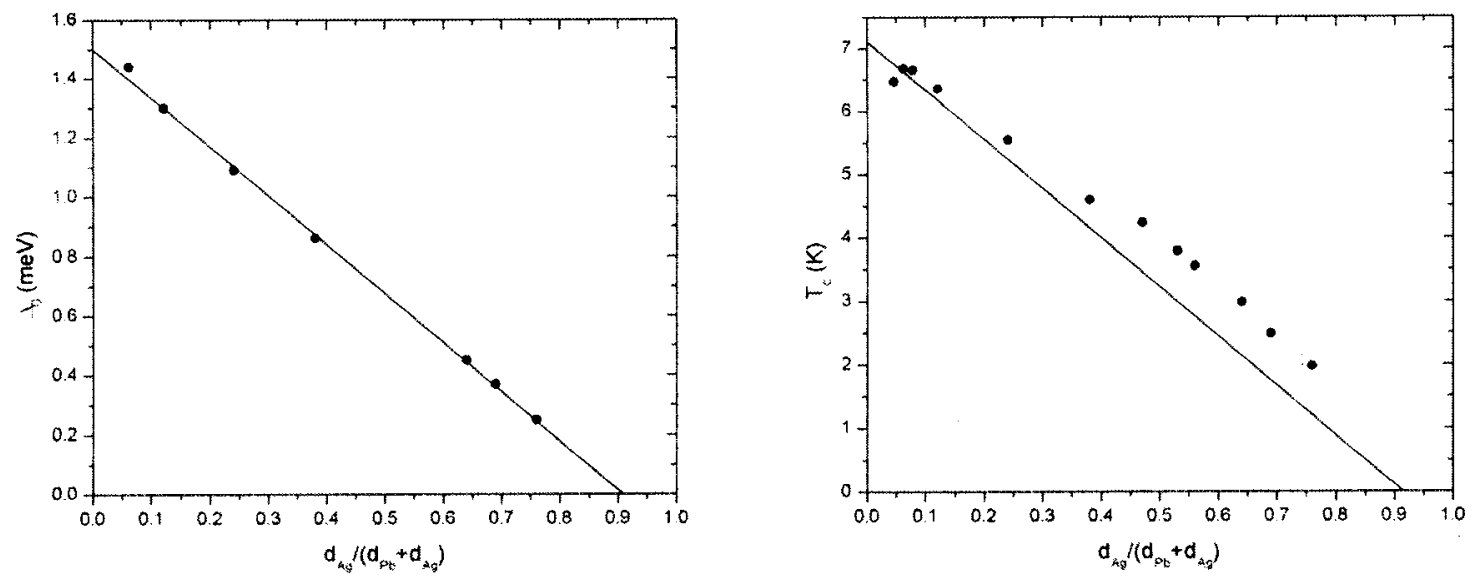

\section{(b) Initial superconducting Pb film + Ag}
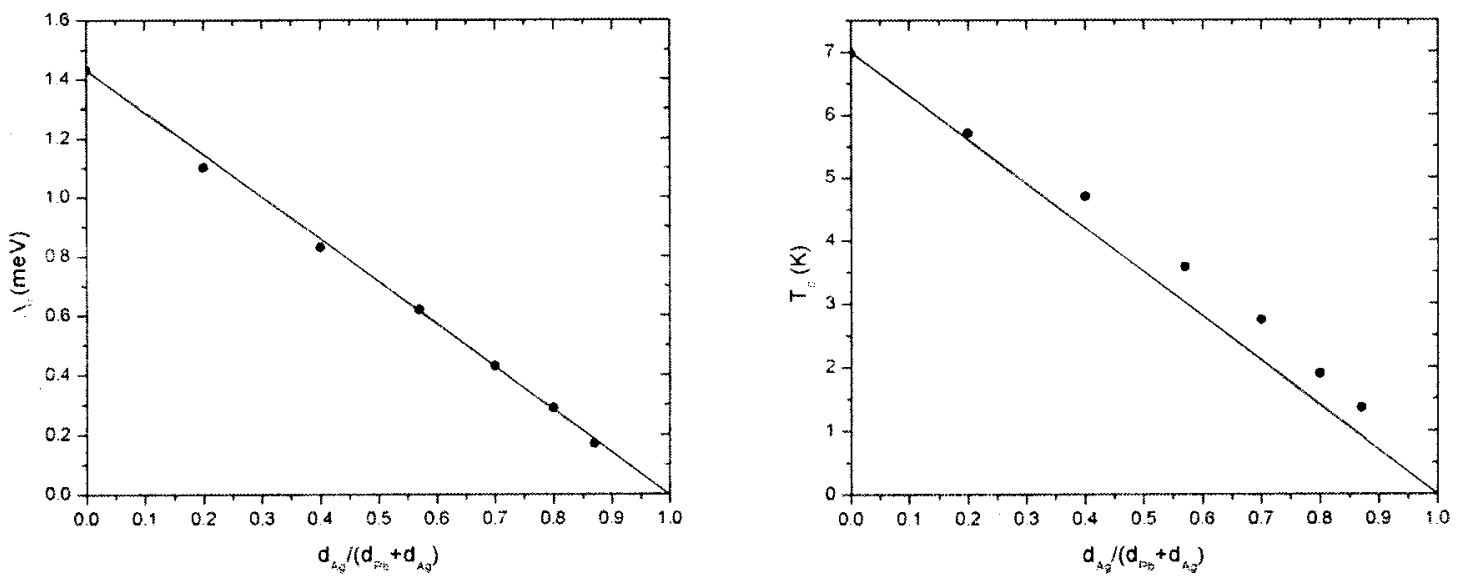

FIG. 7. $\Delta_{0}$ and $T_{c}$ as a function of the parameter $d_{a g} /\left(d_{p b}+d_{a g}\right)$. In case (a) the $\mathrm{Pb}$ film was insulating and the additional Ag induced the superconductivity. In case (b) the initial $\mathrm{Pb}$ film was already superconducting and the additional $\mathrm{Ag}$ reduced $T_{c}$.

measurements are in the Cooper limit comes from the tunneling measurements performed on the same sample. The tunnel junction configuration is such that the Al counter electrode is deposited on the substrate before the granular $\mathrm{Pb}$ is deposited and so in this entire sequence, electrons tunneling into this proximity bilayer are tunneling into the $\mathrm{Pb}$ side of the pair. If we were not in the Cooper limit we would not see an impact of the $\mathrm{Ag}$ in the tunnel measurements. In addition, the Ag would be shunted by the superconducting $\mathrm{Pb}$ and we would not see a decreasing $T_{c}$.

The results of a set of tunneling measurements are shown in Fig. 6. Here we show tunneling $I$ - $V$ curves taken at $1.5 \mathrm{~K}$ for the sequence in Fig. 5. Curve $a$ is for the insulator and is similar to the result in Fig. 3. In this case, with increasing Ag the mean-field transition temperature $T_{\mathrm{mf}}$ decreases, so does the superconducting energy gap. Indeed these curves can be analyzed using standard tunneling analysis techniques ${ }^{5}$ and the $T=0$ energy gap extracted. We have performed this analysis in two cases. In the first case, we have deposited a granular $\mathrm{Pb}$ film and stopped the evaporation while the film is still insulating (following the procedure illustrated in the data of Fig. 5). This was followed by Ag evaporations. In a second experiment, we have deposited enough $\mathrm{Pb}$ to produce a "superconducting" film (approximately curve $j$ in Fig. 2). We then added Ag evaporations to study the effect on an already-superconducting film. The results of the analysis of $\Delta$ and $T_{c}$ in both cases are shown in Fig. 7. There are only very subtle differences in the results of the two experiments. The analysis shown in Fig. 7 in both cases shows the simple relationship between the measured $\Delta$ and $d_{\mathrm{Ag}} /\left(d_{\mathrm{Pb}}+d_{\mathrm{Ag}}\right)$. On the other hand, $T_{c}$ shows some deviation from a linear relationship with $d_{\mathrm{Ag}} /\left(d_{\mathrm{Pb}}+d_{\mathrm{Ag}}\right)$. There is a simple reason for this deviation and that is illustrated in Fig. 8 where we plot the ratio $2 \Delta / k T_{c}$ as a function of $d_{\mathrm{Ag}} /\left(d_{\mathrm{Pb}}+d_{\mathrm{Ag}}\right)$. In both cases, from the data of Fig. 7, it appears that the ratio $2 \Delta / k T_{c}$ is simply changing from the strong coupling value of $\approx 4.8$ to the BCS value of $\approx 3.5 \mathrm{as} \mathrm{Ag}$ is added and the $T_{c}$ is reduced. The value of $\Delta$ used in these analyses is the extrapolated $T=0$ value. This was achieved by determining $\Delta(T)$ and extrapolating to $T=0$ from known behavior. The 


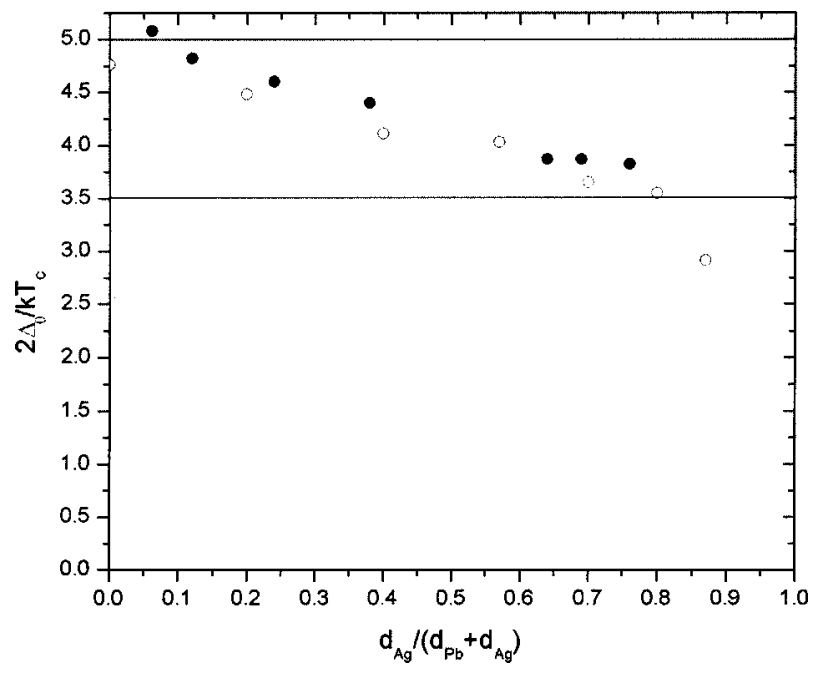

FIG. 8. $2 \Delta_{0} / k T_{c}$ for the two cases illustrated in Fig. 7. Closed circles are for the case of insulating $\mathrm{Pb}$. In addition to reducing $T_{c}$, the addition of $\mathrm{Ag}$ suppresses the strong coupling of $\mathrm{Pb}$ to the weak coupling ratio $2 \Delta_{0} / k T_{c}=3.5$. Within scatter in the data there is no difference in the two cases illustrating that the measurement of $\Delta_{0}$ is the mean-field value.

consistency of this analysis gives us confidence that the proximity effect in the Cooper limit is an appropriate description and the $\mathrm{Pb} / \mathrm{Ag}$ sandwich can be thought of as a homogeneous material insofar as the amplitude $\psi_{0}$ of the order parameter is concerned. Drawing the analogy from Fig. 1 on the overdoped side of the phase diagram then seems appropriate. Naturally then, with increasing $\mathrm{Ag}$, $[N(0) V]_{S+N}$ decreases and $T_{c}$ follows.

\section{SUMMARY}

In summary, we have demonstrated a system that allows us to study in a continuous fashion the crossover from a phase fluctuation dominated superconductor to one that is more traditionally controlled by the amplitude of the order parameter. We have drawn the analogy between the behavior of this system and the high- $T_{c}$ phase diagram. There are differences that should be recognized, but we find the similarities instructive and intriguing. A major difference in the observations is that on the phase fluctuation dominated side of the phase diagram, it is not clear that we can unequivocally define a superconducting transition $T_{c}$. Whether this difference is due to the two-dimensional nature of the current system or whether the analogy is not complete is not currently clear. Nevertheless, strong fluctuations, a '"pseudogap,' and a 'superconducting insulator', are all observed and understood in terms of the decoupling of the grains of the granular system. We do not currently understand the physics of the exponential nature of the resistance in Figs. 2 and 5 but believe it must be related to quantum fluctuations. We think the comparison between our model system and the high- $T_{c}$ superconductors is fascinating and suggest further study.

\section{ACKNOWLEDGMENTS}

We thank S. Kivelson for continued discussions and stimulation. This work was supported by NSF Grant No. DMR9705180.
${ }^{1}$ M. Tinkham, Introduction to Superconductivity (McGraw-Hill, New York, 1975).

${ }^{2}$ V. Emery and S. A. Kivelson, Nature (London) 374, 434 (1995); E. W. Carlson, S. A. Kivelson, V. J. Emery, and E. Manousakis, Phys. Rev. Lett. 83, 612 (1999).

${ }^{3}$ T. Timusk and B. Statt, Reports on Progress in Physics (IOP Publishing, U.K., 1999), Vol. 62.

${ }^{4}$ R. C. Dynes, J. P. Garno, and J. M. Rowell, Phys. Rev. Lett. 40, 479 (1978); H. M. Jaeger, D. B. Haviland, B. G. Orr, and A. M. Goldman, Phys. Rev. B 40, 182 (1989); R. P. Barber, Jr. and R. E. Glover III, ibid. 42, 6754 (1990).

${ }^{5}$ R. P. Barber, Jr., L. M. Merchant, A. La Porta, and R. C. Dynes, Phys. Rev. B 49, 3409 (1994).

${ }^{6}$ A. E. White, R. C. Dynes, and J. P. Garno, Phys. Rev. B 33, 3549
(1986).

${ }^{7}$ A. Truscott, Ph.D. thesis, University of California, San Diego, 1999.

${ }^{8}$ R. C. Dynes, R. P. Barber, Jr., and F. Sharifi, in Ordering Disorder: Prospect and Retrospect in Condensed Matter Physics, edited by Vipin Srivastava, Anil K. Bhatnagar, and Donald G. Naugle, AIP Conf. Proc. No. 286 (AIP, New York, 1994), pp. 96-108.

${ }^{9}$ T. Van Duzer and C. W. Turner, Principles of Superconductive Devices and Circuits (Elsevier, Amsterdam, 1981).

${ }^{10}$ G. Deutscher and P. G. DeGennes, in Superconductivity, edited by R. D. Parks (Marcel Dekker, New York, 1969)

${ }^{11}$ L. Cooper, Phys. Rev. Lett. 6, 689 (1961). 